\title{
Total laparoscopic hysterectomy for large uterine cervical myoma
}

\author{
KENTARO NAKAYAMA*, MAYU TSUKAO*, MASAKO ISHIKAWA, TOMOKA ISHIBASHI, KOHEI NAKAMURA, \\ KAORI SANUKI, HITOMI YAMASHITA, RURIKO ONO, TOSHIKO MINAMOTO and SATORU KYO
}

Department of Obstetrics and Gynecology, Shimane University School of Medicine, Izumo, Shimane 6938501, Japan

Received October 11, 2016; Accepted January 30, 2017

DOI: $10.3892 / \mathrm{mco} .2017 .1217$

\begin{abstract}
Ureterolysis is a surgical method with a high level of difficulty, which may be necessary when performing total laparoscopic hysterectomy (TLH) for large cervical myoma, despite the benign nature of this tumor. The aim of the present study was to introduce techniques that are commonly applied in malignant tumor surgery in order to safely perform TLH for large cervical myoma. Between 2014 and 2016, TLH was performed at the Shimane University Hospital (Izumo, Japan) in 153 patients with benign tumors, including 25 cases with a large uterus (uterine weight $\geq 500 \mathrm{~g}$ ). The surgical methods applied in 3 of these large uterine cervical myoma cases were investigated in detail, including techniques devised by our department. TLH was performed without enucleating myomectomy in all 3 cases; however, all 3 cases required ureterolysis, transection of the anterior layer of the vesicouterine ligament and isolation of the ureter. In conclusion, although radical laparoscopic hysterectomy is commonly performed for cervical cancer at our department, techniques used for malignant tumor surgery may prove useful for benign cases with a high level of difficulty.
\end{abstract}

\section{Introduction}

Compared with laparotomic simple total hysterectomy, total laparoscopic hysterectomy (TLH) is a less invasive procedure that has recently gained popularity in Japan. Although a benign condition, surgery for large myoma of the uterus with extensive adhesions may be challenging, due to the high level of difficulty of the TLH procedure and difficulties with the identification of the uterine artery and ureter, which are mainly attributed to difficulty securing the visual field and operative space and poor uterine mobility (1-3). Among large

Correspondence to: Dr Kentaro Nakayama, Department of Obstetrics and Gynecology, Shimane University School of Medicine, 89-1 Enyacho, Izumo, Shimane 6938501, Japan

E-mail:kn88@med.shimane-u.ac.jp

*Contributed equally

Key words: total laparoscopic hysterectomy, large cervical leiomyoma, vesicouterine ligament anterior layer myomas of the uterus, cervical myoma requires skilled techniques for dissecting the cervix, and surgery is considered the most difficult among TLH procedures for benign conditions. Our department devised various techniques to safely treat these difficult cases with surgery. TLH was applied to large cervical myoma in 3 patients. We herein report these cases in detail, including a description of the techniques devised by our department.

\section{Case presentation}

TLH was introduced in the Department of Obstetrics and Gynecology, Shimane University Hospital (Izumo, Japan) in July, 2014. By June, 2016, a total of 230 patients had undergone this procedure (Fig. 1). Of the 230 cases, 200 were benign and 30 were malignant, including those treated with laparoscopic radical hysterectomy. The tumor was a giant myoma (uterine weight, $\geq 500 \mathrm{~g}$ ) in 25 of the 200 benign cases. There were 3 cases of cervical myoma of the uterus and these are presented in detail below (Table I).

Case 1. The patient was a 51-year-old woman, gravida 2, para 2. On magnetic resonance imaging (MRI) examination, a cervical myoma measuring 108x103x90 mm (depth, width and height, respectively) was identified on the left side over the posterior wall of the uterine cervix. The uterus was enlarged to $155 \times 103 \times 102 \mathrm{~mm}$, and the uterine fundus reached $5 \mathrm{~cm}$ cranial to the promontory (Fig. 2A). During surgery, the uterus reached right below the camera port in the umbilical region, and the round ligament was markedly shortened (Fig. 2B). Although a uterine manipulator was used, uterine mobility was poor. Thus, the uterine round ligament, anterior lobe of the broad ligament and infundibulopelvic ligament were first dissected to secure uterine mobility, followed by dissecting the bilateral pelvic sidewall triangles and identification of the uterine artery and ureter. To dissect the contralateral pelvic sidewall triangle, the operator stood on the ipsilateral side to approach it. To mobilize the cervix, the anterior layer of the left vesicouterine ligament was resected. A vaginal tube (Vagi-Pipe ${ }^{\circledR}$, Hakko Medical Co., Ltd., Chikuma, Japan) was inserted and the vaginal wall was cut. Removal of the uterus through the vagina was attempted, but it was difficult due to poor extensibility of the vaginal wall. The camera port in the umbilical region was incised to expand to $3 \mathrm{~cm}$, and the uterus was cut into small pieces while being distracted using a Martin forceps and collected through the port. The blood loss 
was significant $(1,380 \mathrm{ml})$ and the hemoglobin level decreased to $5.6 \mathrm{~g} / \mathrm{dl}$ immediately after the surgery, for which $400 \mathrm{ml}$ blood transfusion were required. The cause for the large volume of blood loss was cutting the very large uterus into smaller pieces in order to remove it from the abdominal cavity. The total duration of this procedure was $94 \mathrm{~min}$ and bleeding from the transected vaginal stump continued during that time. The overall operative time was $285 \mathrm{~min}$ and the weight of the excised uterus was $1,100 \mathrm{~g}$ (Fig. 2B).

Case 2. The patient was a 51-year-old woman, gravida 3, para 2 (2 caesarean sections). On MRI examination, a cervical myoma measuring $60.3 \times 52.4 \times 57.8 \mathrm{~mm}$ was present on the left side over the posterior wall of the uterine cervix, and a cervical myoma measuring $6.3 \times 31.7 \times 24.8 \mathrm{~mm}$ was present at the median line of the anterior wall. The uterus was increased in size to $107 \times 76 \times 54 \mathrm{~mm}$ and the uterine fundus reached $2.6 \mathrm{~cm}$ cranial to the promontory (Fig. 3A). During surgery, the uterine mobility was favorable and the pelvic sidewall triangle was normally dissected followed by identification of the uterine artery and ureter (Fig. 3B). To mobilize the cervix, the anterior layer of the left vesicouterine ligament was dissected. A Vagi-Pipe was inserted to cut the vaginal wall and the uterus was removed through the vagina. The blood loss was small, the operative time was $145 \mathrm{~min}$ and the weight of the excised uterus was $501.5 \mathrm{~g}$ (Fig. 3B).

Case 3. The patient was a 60 -year-old woman, gravida 3 para 2. On MRI examination, a cervical myoma measuring 107x70x88 mm was identified on the left side over the posterior wall of the uterine cervix, the uterus was increased in size to $110 \times 105 \times 88 \mathrm{~mm}$ and the uterine fundus was at the level of the promontory (Fig. 4A). During surgery, uterine mobility was somewhat poor; thus, the round ligament and anterior lobe of the broad ligament were first dissected followed by dissection of the pelvic sidewall triangle and identification of the uterine artery and ureter (Fig. 4B). The anterior layer of the left vesicouterine ligament was dissected first in order to mobilize the cervix. A Vagi-Pipe was inserted to cut the vaginal canal and the uterus was transvaginally cut into small pieces and removed. The blood loss was $400 \mathrm{ml}$, the operative time was $192 \mathrm{~min}$ and the weight of the excised uterus was $605 \mathrm{~g}$ (Fig. 4B).

Basic TLH procedure in our department. In the lithotomy position under general anesthesia, after placing a uterine manipulator, the first port was inserted through the $12-\mathrm{mm}$ diameter camera port prepared in the umbilical region and pneumoperitoneum was applied. Ports with diameters of 5 , 12 and $5 \mathrm{~mm}$ were placed in the left and right iliac fossae and the center between the bilateral iliac fossae, respectively, arranging the ports in a diamond pattern setting the apex to the umbilical region. The operator and first assistant stood on the left and right sides of the patient, respectively, and the second assistant operated the manipulator. The unilateral pelvic sidewall triangle (the region surrounded by the round ligament, infundibulopelvic ligament and posterior lobe of the broad ligament) was dissected. The posterior lobe of the broad ligament, paravesical space and pararectal spaces of Okabayashi and Latzko were exposed. The uterine artery and ureter were identified, and the uterine artery was ligated with absorbable thread, coagulated and cut. Dissection of the uterine artery and ureter was advanced, and the ureter was dissected up to the ureteral tunnel. These procedures were similarly applied on the opposite side. Subsequently, the round ligament, anterior lobe of the broad ligament, ligamentum ovarii proprium (infundibulopelvic ligament), posterior lobe of the broad ligament and uterosacral ligament were coagulated and cut. The peritoneum of the vesicouterine pouch was incised and dissected to expose the pouch. The parametrium was coagulated and cut. The manipulator was removed, a Vagi-Pipe was inserted along the vaginal fornix and the vaginal wall was cut along the Vagi-Pipe. The excised specimen was extracted through the vagina, and the vaginal stump was sutured and closed with absorbable thread. The central peritoneum was continuously sutured and closed with absorbable thread. Following irrigation and hemostasis, an antiadhesive agent was applied to the wound, the laparoscopic surgery was completed and the abdominal cavity was closed.

Devices used for the treatment of large cervical myomas in our department. The following 3 points were devised to treat the 3 cases of large cervical myoma in our department, which enabled completion of TLH.

Dissection of the anterior layer of the vesicouterine ligament (Fig. 5). The anterior layer of the vesicouterine ligament was dissected in all 3 cases. In normal TLH, the ureter is separated from the uterine cervix when dissecting the parametrium, during which the cut vaginal wall may be identified, but this conventional dissection of the parametrium cannot be applied in cases of cervical myoma, and identification of the positional association between the cervix and ureter is difficult. Thus, the anterior layer of the vesicouterine ligament was dissected applying techniques usually reserved for malignant tumor surgery, which enabled safely separating the ureter from the cervix and cutting the vaginal wall.

When dissecting the anterior layer of the vesicouterine ligament in laparotomic surgery, Cooper forceps are passed through the ureteral tunnel and used to cut the anterior layer of the vesicouterine ligament (Fig. 5A). Performed through the same approach in laparoscopic surgery, but with the added major advantage of laparoscopy, which is acquisition of an extended visual field, the connective tissue on the cranial side of the ureteral tunnel may be cut and the sheath containing the ureteral tunnel may be entirely dissected towards the caudal side without cutting the vesicouterine ligament (Fig. 5B). Using this technique, thermal damage of the ureter near the anterior layer of the vesicouterine ligament may be prevented.

Positioning of the operator to access the pelvic sidewall triangle. In conventional TLH, the operator stands on the left side of the patient and dissects the bilateral pelvic sidewall triangles. However, to dissect the right pelvic sidewall triangle in large myoma cases is difficult, as the forceps and devices interfere with the enlarged uterus when using the left-sided approach. Thus, for large myomas, the operator stands on the ipsilateral side of the pelvic sidewall triangle to be dissected.

Dissection of the round ligament, anterior lobe of the broad ligament of the uterus and ligamentum ovarii proprium 
Table I. Three cases of large cervical myoma.

\begin{tabular}{lccc}
\hline Clinicopathological parameters & Case 1 & Case 2 & Case 3 \\
\hline Age, years & 51 & 51 & 59 \\
BMI, $\mathrm{kg} / \mathrm{m}^{2}$ & 26.6 & 23.6 & 28.5 \\
Size of cervical myoma, mm & $108 \times 103 \times 90$ & $60.3 \times 52.4 \times 57.8$ & $107 \times 70 \times 88$ \\
(depth $\mathrm{x}$ width $\mathrm{x}$ height) & & $26.3 \times 31.7 \times 24.8$ & $110 \times 105 \times 88$ \\
Uterine size, mm & $155 \times 103 \times 102$ & $107 \times 76 \times 54$ & Left-posterior wall \\
(depth x width $x$ height) & & & Intramuscular layer-subseroma \\
Location & Left-posterior wall & Median of the anterior wall & 192 \\
Type & Intramuscular layer & Intramuscular layer & 400 \\
Operative time, min & 285 & 145 & None \\
Blood loss, ml & 1,380 & 0 & None \\
Blood transfusion, $\mathrm{ml}$ & 400 & None & None \\
Switch to laparotomy & None & & \\
\hline
\end{tabular}

BMI, body mass index.

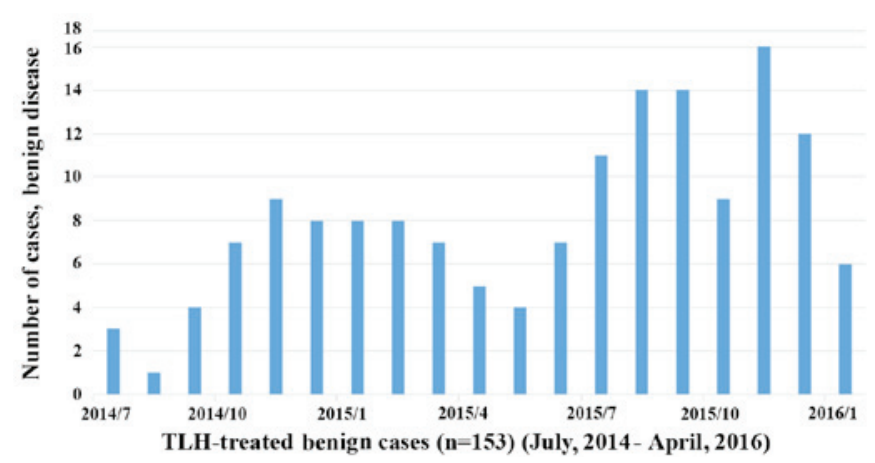

Figure 1. TLH-treated benign cases $(n=153)$ at the Shimane University Hospital between July, 2014 and April, 2016. TLH, total laparoscopic hysterectomy.

(infundibulopelvic ligament). When uterine mobility is favorable, a uterine manipulator is inserted, the uterus may be freely moved, tension may be readily loaded on the ligaments supporting the uterus, and the pelvic sidewall triangles may be readily dissected, followed by dissection of the uterine artery and ureter. However, uterine mobility is poor in cases with large uterine myomas, although a manipulator is inserted, and dissecting the pelvic sidewall triangle may be difficult. Thus, to achieve uterine mobility, the round ligament, ligamentum ovarii proprium (infundibulopelvic ligament) and anterior lobe of the broad ligament of the uterus are dissected first.

\section{Discussion}

LH was initially reported by Reich et al in 1986 and has been rapidly spreading in Japan (4). TLH was introduced in our department in 2014 and is performed using the standardized procedure. The time required for the surgery has been shortened and the number of cases treated with TLH has increased. In addition, we were able to apply TLH to more difficult cases of large myomas and adhesions. Our department introduced laparoscopic radical surgery of endometrial cancer in November, 2014 and laparoscopic radical hysterectomy of cervical cancer in May, 2015, performed in 27 and 7 patients, respectively, between July, 2014 and July, 2016. TLH was safely performed in 3 cases of large cervical myoma, and this may have been made possible due to the dissection of the anterior layer of the vesicouterine ligament utilizing the techniques usually reserved for malignant tumor surgery. In Japan, it is recommended that a physician certified in gynecological endoscopic techniques and a physician specialized in gynecological oncology cooperate to introduce laparoscopic surgery for malignant diseases, but not all institutions have both types of physicians. Our department only has physicians specialized in gynecological oncology, who introduced TLH. Physicians specialized in gynecological oncology are familiar with surgery for malignant tumors, ureterolysis and dissection of the pelvic blood vessels; thus, they may rapidly improve endoscopic surgical techniques through short-term training on endoscopic surgery. Based on the speed of the spread of TLH and introduction of laparoscopic surgery for malignant tumors in our department, the results indicate that physicians specializing in gynecological oncology should shift to endoscopic surgery as soon as possible in Japan.

There are several previous reports on TLH for large myoma of the uterus (1-3); however, to the best of our knowledge, TLH for large cervical myoma has not been previously reported. Thus, this may be a useful report, despite the limited number of cases. In addition, to the best of our knowledge, there has been no previous report on the method by which the anterior layer of the vesicouterine ligament is dissected to treat large cervical myomas. Further cases must be accumulated to investigate its usefulness.

In addition to the methods devised by our department, the usefulness of placing an upper abdominal port, preceding enucleating myomectomy, and the use of an electric morcellator for the treatment of large uterine myomas has been previously reported (5), as has the usefulness of retrograde 

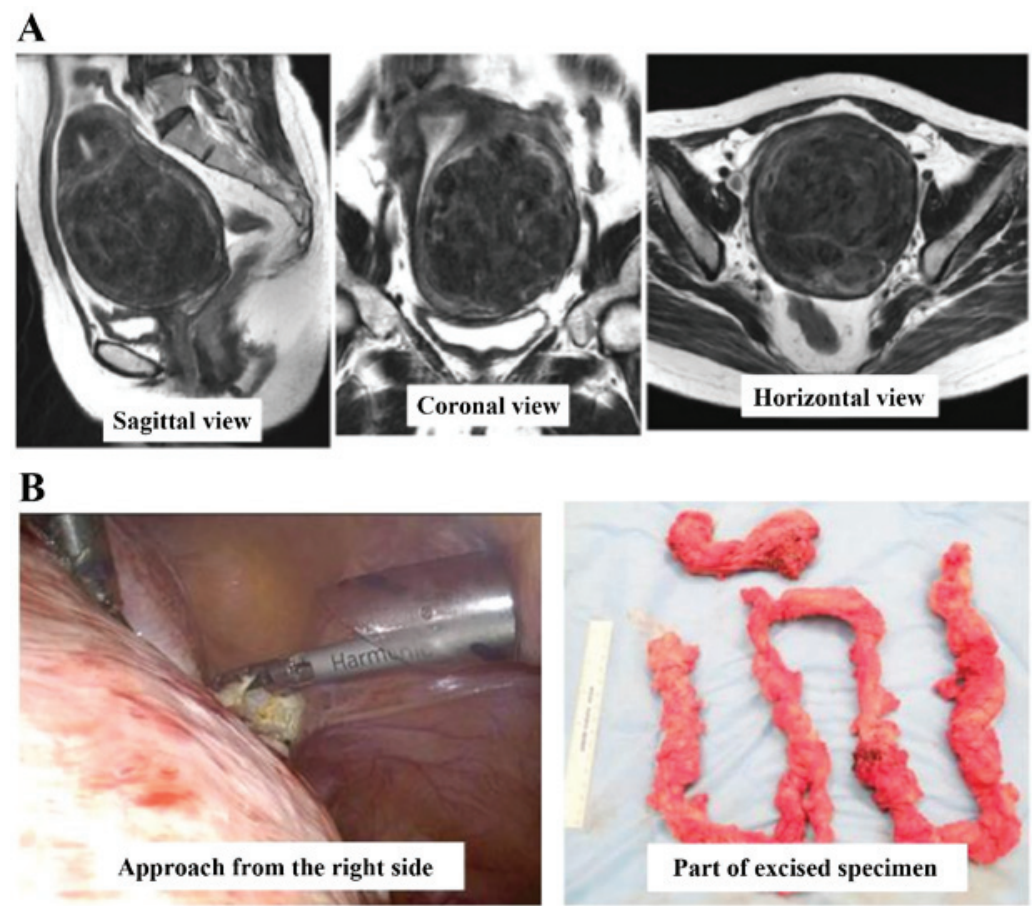

Figure 2. Case 1. (A) Plain pelvic magnetic resonance imaging (T2-weighted images). Left panel, sagittal view; central panel, coronal view; and right panel, horizontal view. (B) Surgical findings. Left panel, laparoscopic approach from the right side; right panel, part of the excised specimen.

$\mathbf{A}$

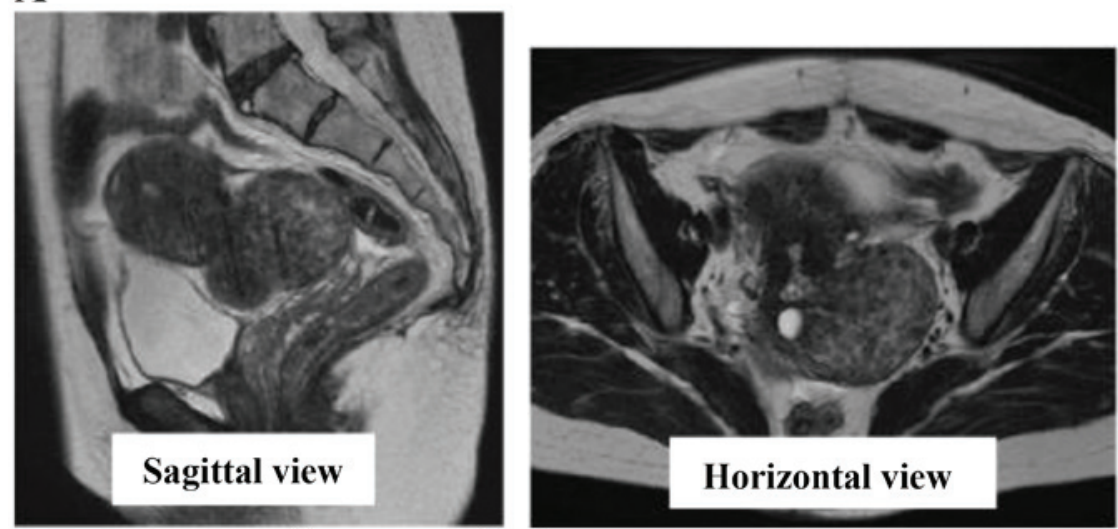

B

Laparoscopic view
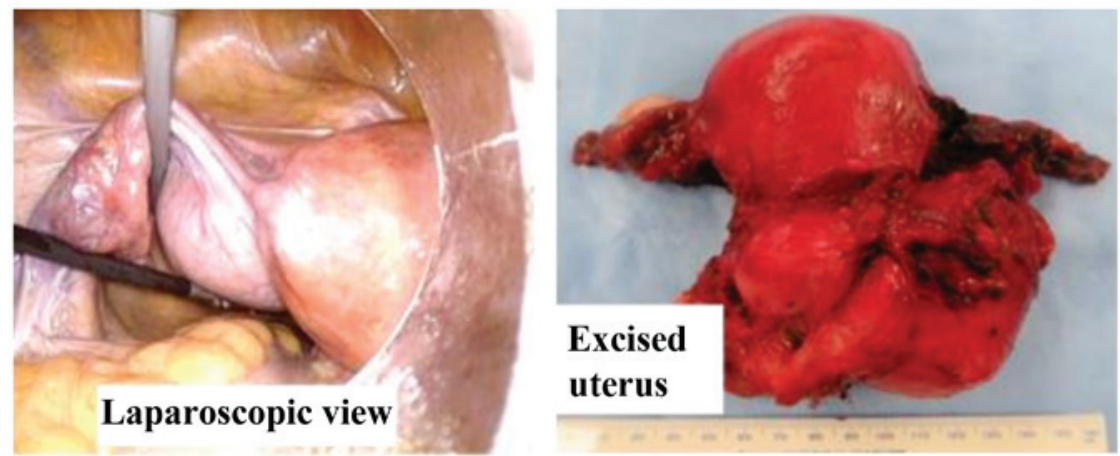

Figure 3. Case 2. (A) Plain pelvic magnetic resonance imaging (T2-weighted images). Left panel, sagittal view; right panel, horizontal view. (B) Surgical findings. Left panel, laparoscopic view; right panel, excised uterus.

TLH for the treatment of advanced adhesions and cases with an unclear cervical region $(6,7)$. Regarding large myomas of the uterus, TLH is applicable even for uterine myomas weighing $>1,000 \mathrm{~g}$. It has been reported that the rate of switch to laparotomy was $4.2-9.7 \%$, a small incision in the abdominal wall was necessary to extract the specimen in $21 \%$ of the 

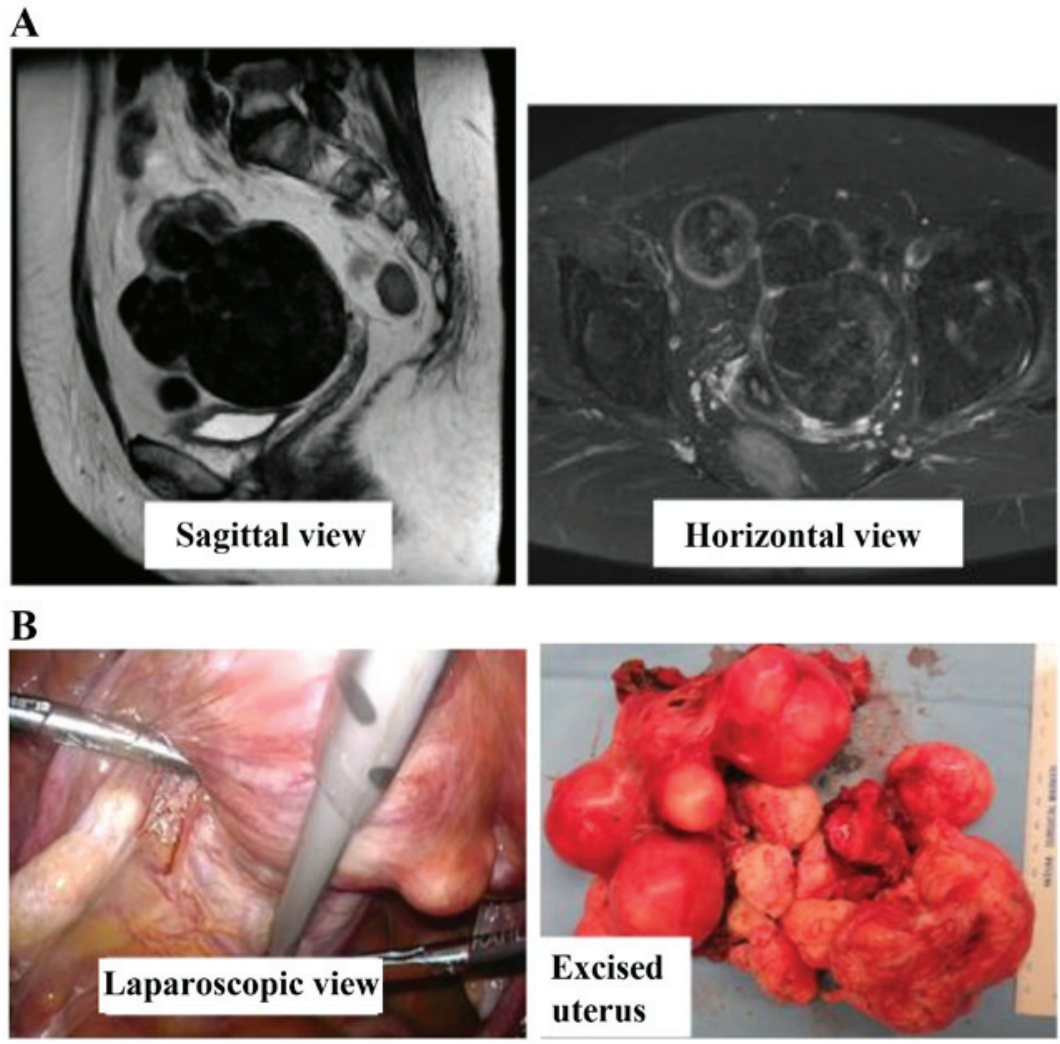

Figure 4. Case 3. (A) Plain pelvic magnetic resonance imaging (T2-weighted images). Lt. panel showed sagittal view. Rt. panel shoed horizontal view. (B) Surgical findings. Left panel, laparoscopic view; right panel, excised uterus.

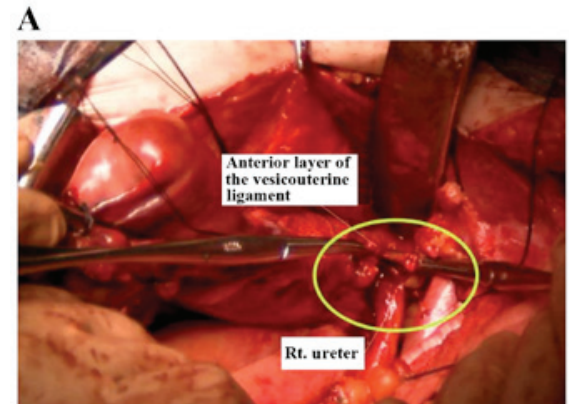

B

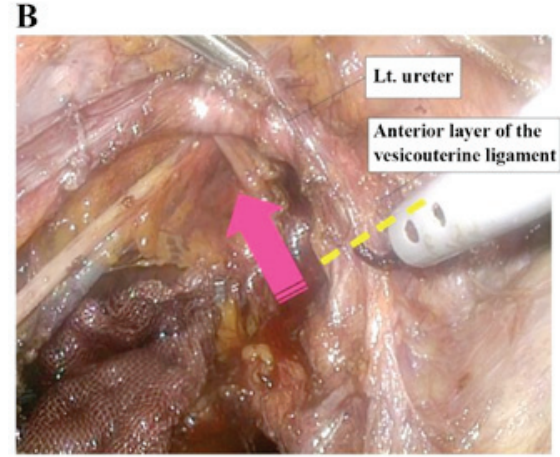

Figure 5. Dissection of the anterior layer of the vesicouterine ligament by (A) laparotomic surgery and (B) laparoscopic surgery.

cases, and the incidence of complications was $2.8-11.4 \%$ (3). Our department has performed TLH in 25 cases with a uterine weight of $>500 \mathrm{~g}$, with no reported ureteral damage or switch to laparotomy (data not shown).
In case 1 , although the myoma was sizeable and the excised specimen weighed $1,100 \mathrm{~g}$, TLH was completed without complications. However, $94 \mathrm{~min}$ were required to extract the specimen, during which bleeding from the vaginal stump continued and blood transfusion was required. Based on this experience, the vaginal stump is sutured in advance when removing a large myoma specimen is likely to be time-consuming, and the umbilical port is extended by incising the region by $3 \mathrm{~cm}$ to extract the specimen. No blood transfusion was required in any of the large myoma cases managed thereafter in our department.

The use of an electric morcellator to remove specimens is associated with the risk of scattering malignant tissue not preoperatively diagnosed, parasitic myomatosis, postoperative infection and pain. An electric morcellator is not used in our department; the tissue is cut into pieces using a Cooper forceps and transvaginally collected through the umbilical port. The abovementioned risks may be avoided using a morcellator in a bag; however, the possibility of perforating the bag has been reported. Surgery may be safely performed using a large isolation bag as reported in another study, but the use of this method was off-label and further investigation is required (8).

TLH for large cervical myoma was safely completed by dissecting the anterior layer of the vesicouterine ligament, by the operator approaching the pelvic sidewall triangles from the respective ipsilateral side, and by dissecting the round ligament, anterior lobe of the broad ligament of the uterus and ligamentum ovarii proprium in advance. Although not all facilities are certified to perform laparoscopic radical hysterectomy and this procedure is not widely used in Japan, 
difficult cases of benign tumors may be handled by applying procedures usually reserved for malignant tumor surgery. Thus, the acquisition of skills in laparoscopic radical hysterectomy is crucial.

\section{References}

1. Brummer TH, Jalkanen J, Fraser J, Heikkinen AM, Kauko M, Mäkinen J, Seppälä T, Sjöberg J, Tomás E and Härkki P: FINHYST, a prospective study of 5279 hysterectomies: Complications and their risk factors. Hum Reprod 26: 1741-1751, 2011.

2. Ceccaroni M, Roviglione G, Pesci A, Quintana S, Bruni F and Clarizia R: Total laparoscopic hysterectomy of very enlarged uterus $(3030 \mathrm{~g})$ : Case report and review of the literature. Wideochir Inne Tech Maloinwazyjne 9: 302-307, 2014.

3. Uccella S, Cromi A, Serati M, Casarin J, Sturla D and Ghezzi F: Laparoscopic hysterectomy in case of uteri weighing1 kilogram: A series of 71 cases and review of the literature. J Minim Invasive Gynecol 21: 460-465, 2014
4. Reich H, Decaprio J and McGlynn F: Laparoscopic hysterectomy. J Gynecol Surg 5: 213-216, 2009.

5. Takamatsu S, Matsubara M, Onuma T, Tsuji T, Tajima K and Shimada H: Total laparoscopic hysterectomy for large uteri weighing more than 500 grams at a single institution. Jap J Gynecol Obst Endosc 30: 169-176, 2014

6. Hirata G, Yumori A, Naruke Y, Furuno A, Kitagawa M, Katayama K, Ohshima A, Murase M, Yoshida H and Hirahara F. Analysis of 276 cases of total laparoscopic hysterectomy. Jap J Gynecol Obst Endosc 29: 408-412, 2014

7. Kuramori R, Masumoto T, Hashimoto Y, Saeki A, Oku H, Kuno A and Ikuma K: Surgical technique and usefulness of Retrograde approach to patients with unclear uterine cevixes and with severe adhesion in a cul-de-sac. Jap J Gynecol Obst Endosc 27: 272-277, 2011

8. Cohen SL, Einarsson JI, Wang KC, Brown D, Boruta D, Scheib SA, Fader AN and Shibley T: Contained power morcellation within an insufflated isolation bag. Obstet Gynecol 124: 491-497, 2014. 